\title{
Microorganisms of the toe web and their importance for erysipelas of the leg
}

\section{Dominik-Patrick Müller', Reinhard Hoffmann², Julia Welzel'}

(1) Department of Dermatology and Allergology, General Hospital Augsburg (2) Institute for Laboratory Medicine, Microbiology and Environmental Hygiene, General Hospital Augsburg

\section{Summary}

Background: There has been a steady increase of the incidence of erysipelas in Germany in the recent past. Affected patients also often show defects in the cutaneous barrier caused by microorganisms. The aim of this non-interventional case-control study was to investigate a possible interrelationship between interdigital tinea pedis and bacterial toe web (interdigital space) infections and erysipelas of the leg, as well as a potential interaction among the microorganisms themselves.

Material and methods: The patient population contained 150 people equally distributed among three groups, one retrospective and one prospective erysipelas group $(E G=r E G+p E G)$ plus one control group $(K G)$.

Results: $51 \%$ of the patients with erysipelas and $32 \%$ of the control group suffered from interdigital tinea pedis. There was a significant association between interdigital tinea pedis and the recurrence rate of erysipelas, but not with erysipelas itself. Staphylococcus aureus (EG: 30.34 \%; KG: $3.23 \%$ ) and non-pathogenic aerobic bacteria were significantly associated with erysipelas in each statistical analysis. Staphylococcus aureus showed a positive, the non-pathogenic aerobic germs (EG: 28,09\%; KG: 80,65\%) a negative association.

Conclusions: This study demonstrates an association between the microorganisms of the toe web and erysipelas of the leg. Erysipelas itself is influenced to a great extent by the bacterial flora, while its recurrence relates more to interdigital tinea pedis.

\section{Introduction}

While the incidence of erysipelas in Germany continuously decreased in the last half of the $20^{\text {th }}$ century, during the last two decades a significant increase in incidence as well as a change in predilection sites shifting from the face to the leg has been observed [1-4]. Most patients with erysipelas are elderly and suffer from multiple co-morbidities including diabetes mellitus, arterial hypertension, obesity, peripheral arterial occlusive disease, and onychomycosis as well as interdigital tinea pedis, the latter being considered as one of the most important risk factors in the pathogenesis of erysipelas [5].
The aim of this study was to find out whether interdigital tinea pedis has an influence on erysipelas and to what extent bacterial colonization of the toe web is associated with both interdigital tinea pedis and erysipelas.

\section{Patients and methods}

\section{Patient collective}

The patient cohort $(\mathrm{n}=150)$ was equally distributed among three groups $(n=50)$. The retrospective erysipelas collective included patients who had been treated for erysipelas of the leg in our clinic between January 2011 and January 2012. 
The selection was random and without prior inspection of the patients' medical files.

The acquisition of the prospective groups (prospective erysipelas group and control group) was performed in our clinic between July 2012 and July 2013. Patients were admitted to the study collective after detailed information and informed consent. The diagnostic and non-interventional study was without influence on the treatment of patients. The evaluation of data was strictly anonymous.

The erysipelas group $(n=100)$ consisted of a retrospective and a prospective part. The only inclusion criterion was the presence of erysipelas of the leg. Results of $\mathrm{KOH}$ examination were available for all patients. Bacterial culture data was not available for nine patients in the retrospective group, and two in the prospective group.

The control group consisted of 50 patients with a facial skin cancer and a negative medical history for erysipelas. Results of $\mathrm{KOH}$ examination were available for all patients of the control group. Culture results were available in 31 cases.

\section{Methods of investigation}

Fungal toe web infections were identified by $\mathrm{KOH}$ examination. Sampling points were disinfected before skin scrapings were obtained. Samples pre-treated with $\mathrm{KOH}$ solution and methylene blue before being analyzed microscopically for spores and fungal hyphae. Although dermatophytes cannot be identified specifically in mycological native preparations, due to the basis of our own clinical experience a positive native preparation obtained from the toe web represents, as a general rule, dermatophytes.

In a study performed in our clinic by Rothmund et al. comparing six different diagnostic methods for the proof of onychomycosis, this method of investigation had a sensitivity of $74.4 \%$ and a specificity of $76.2 \%$ [6].

In the context of this study we compared the results of $\mathrm{KOH}$ examination of ten randomly selected samples with the result of polymerase chain reaction (PCR). A 184 bp-segment of the hypervariable V4-domain of the small ribosomal subunit of the 18S-rRNA gene was amplified. DH1L and DH1R were chosen as primer. Identification of DNA fragments was performed with agarose gel electrophoresis. Pan-dermatophyte PCR using the primers panDER-1 and panDER-2 was performed subsequently. In case of a positive dermatophyte result, further classification for Trichophyton mentagrophytes was performed with the primers Tment-F and Tment- $\mathrm{R}$ and for Trichophyton rubrum using the primers Trub-1 and Trub-2. A multiplex PCR was performed for evaluating the quality of isolated DNA.

Blood agar, chocolate agar, MacConkey agar and thioglycollate broth were used as culture media for toe web specimens. The incubation time was 48 hours at $36 \pm 1{ }^{\circ} \mathrm{C}$.
Differentiation followed standard microbiological methods. The combination of at least two of the following bacteria corynebacteria, coagulase-negative staphylococci and/or $\alpha$-hemolytic streptococci - was identified as non-pathogenic aerobic bacteria by the laboratory. A single organism was identified separately. In order to avoid statistical bias, samples were sent to the microbiological laboratory without diagnosis or patient information.

\section{Statistics}

Data obtained were analyzed for arithmetic mean, standard deviation, absolute and relative frequency distribution as well as specificity and sensitivity. Specificity indicates the probability of a negative result in a non-infected patient [7]. Sensitivity shows the percentage of positive results in all truly infected patients $[8,9]$. PCR, as mentioned above, served for estimation of both sensitivity and specificity. Bivariate two-sided Fisher's exact test $(\mathrm{p} \leq 0.001$, highly significant; $\mathrm{p} \leq 0.01$, very significant; $\mathrm{p} \leq 0.05$, significant), odds ratio as well as logistic regression approach served for statistical evaluation of a possible association between erysipelas, interdigital tinea pedis and bacterial colonization of the interdigital space (significance level $\alpha=0.05$ ). Logistic regression, a multivariate method, was used to reduce confounders in order to obtain more precise results $[9,10]$.

\section{Results}

The average age of the erysipelas collective was $67 \pm 16.8$ years and of the control group, $73.2 \pm 9.4 .40 \%$ of the patients $(n=60)$ were women. In $93 \%$ of the cases erysipelas affected the lower leg. $51 \%$ of the patients with erysipelas, but only $10.7 \%$ of the patients of the control group suffered from interdigital tinea pedis. The Fisher exact test revealed a statistically significant association between interdigital tinea pedis and both erysipelas $(\mathrm{p}=0.0363$; OR 2.21) and relapses $(\mathrm{p}=$ 0.0091; OR 2.99). Moreover, a very significant association was found between interdigital tinea pedis and maceration of the toe web $(p=0.0019)$. Validity of $\mathrm{KOH}$ examination was verified by PCR showing a specificity of $66.7 \%$ and a sensitivity of $71.4 \%$. Results of the microbiological analysis of the nine bacteria having the lowest p-value are shown in Figure 1.

In addition to bivariate analysis, multivariate logistic regression was employed. Both the most significant bacteria and interdigital tinea pedis were considered. This statistical approach aimed to determine whether bacteria exclusively, interdigital tinea pedis exclusively or both have a statistically significant influence on erysipelas and/or its recurrence. Moreover, this method was designed to avoid or minimize a potential bias by interference of different influence parameters. Multivariate analysis revealed that non-pathogenic aerobic 


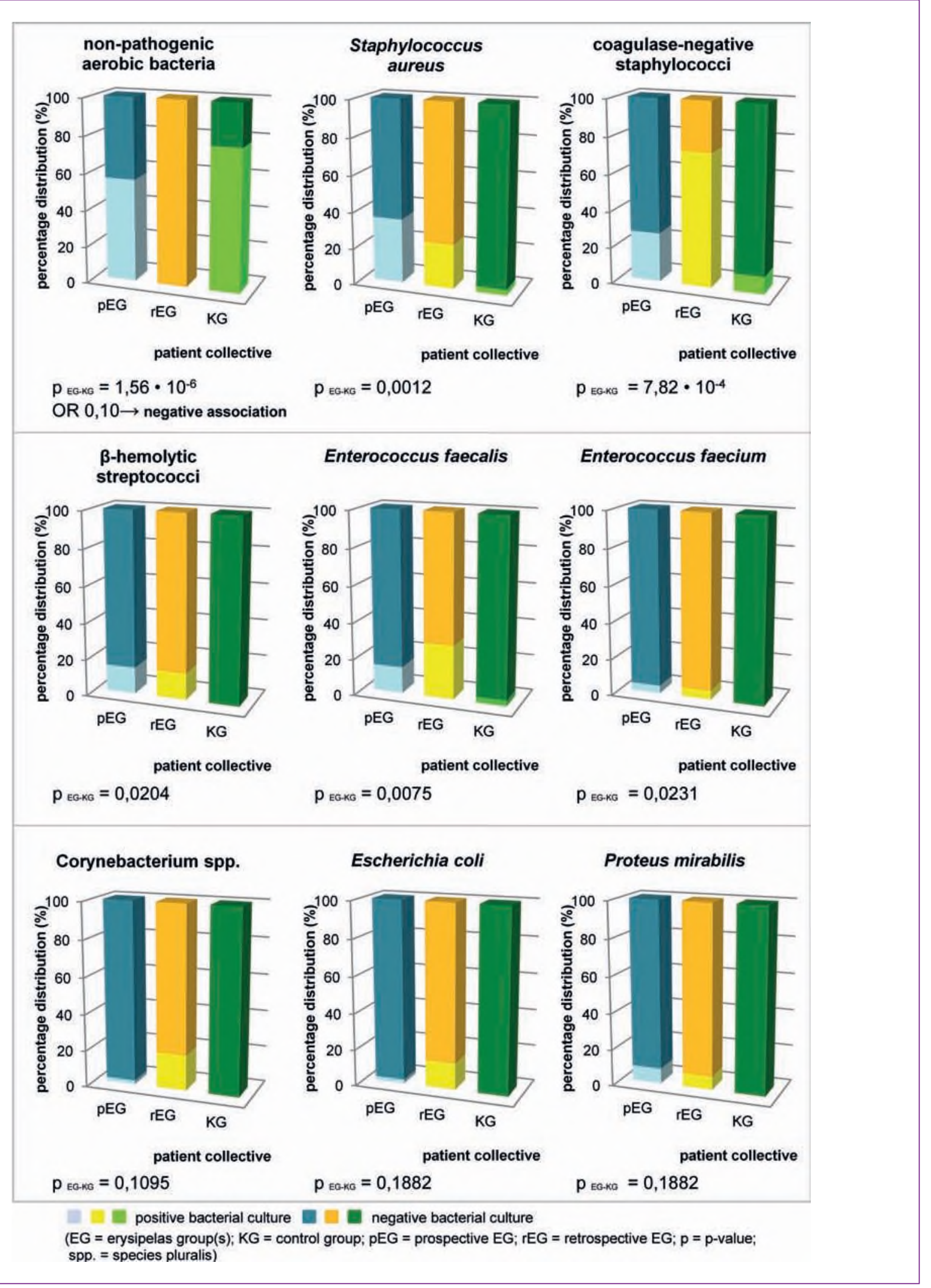

Figure 1 Frequency distribution of positive and negative culture results for (almost) significant bacteria in each single group plus results of the Fisher's exact test (null hypothesis: equal distribution of bacteria in both erysipelas and control group). 


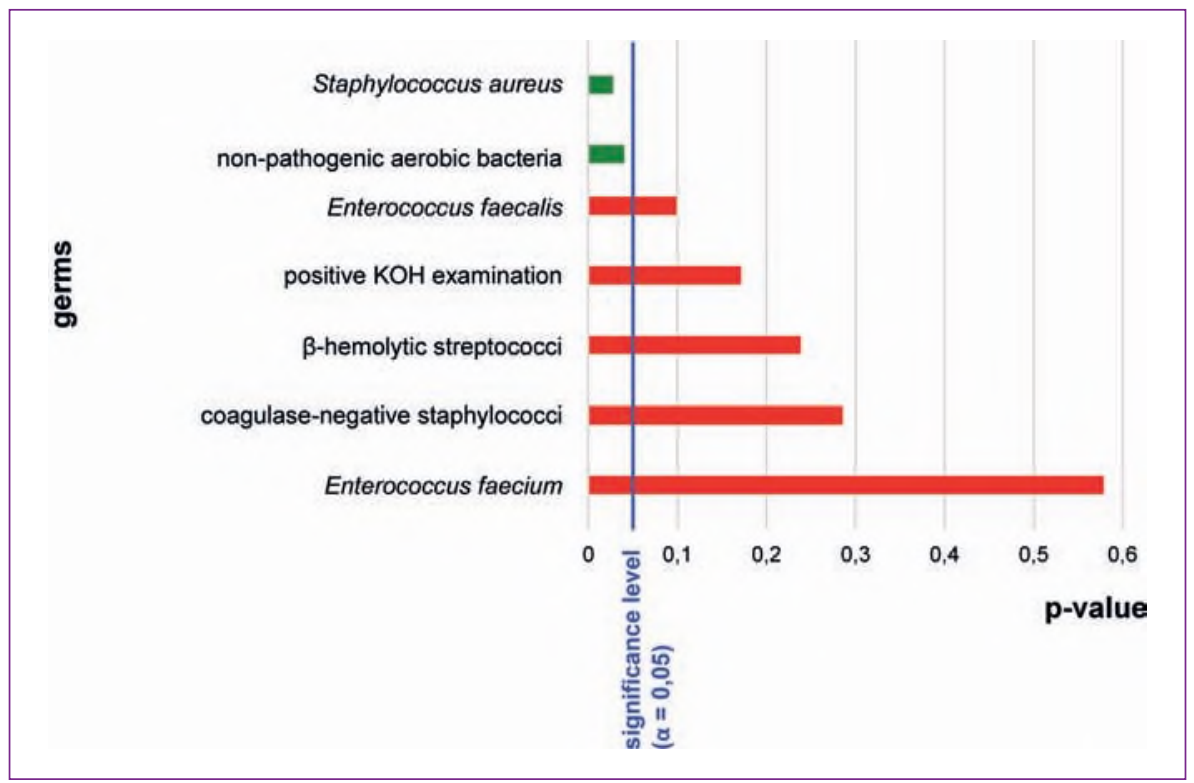

Figure 2 Logistic regression analysis of the six most significant bacteria and $\mathrm{KOH}$ examination (including all groups) in order to exclude confounding. germs ( $\mathrm{p}=0.0406$; OR 0.25; negative association) and Staphylococcus aureus ( $\mathrm{p}=0.0276)$ were statistically significant associated with erysipelas (Figure 2). Only interdigital tinea pedis showed a statistically significant association with recurrence of erysipelas $(\mathrm{p}=0.0475)$.

\section{Discussion}

The influence of interdigital tinea pedis on erysipelas of the leg has been controversially discussed. The study of Roujeau et al. stressed its crucial importance showing a significant association between erysipelas of the leg and interdigital tinea pedis which could be effectively treated [11]. Other authors consider interdigital tinea pedis to be one among many possible risk factors [12]. According to the findings of Björnsdóttir et al. [13] interdigital tinea pedis only appears to be a significant risk factor when bacteria are not taken into account. Our study supports this view. Bivariate statistical evaluation revealed a significant association between erysipelas and interdigital tinea pedis $(p=0.036)$. In contrast to this, multivariate analysis indicated that bacteria and not interdigital tinea pedis are the main risk factor for erysipelas, which is in accordance with the results of Björnsdóttir et al. [13].

Analysis of the entire microbial flora concerning its influence on the recurrence rate of erysipelas reached a different conclusion. Bivariate analysis revealed a statistically significant association between relapses and interdigital tinea pedis ( $\mathrm{p}=0.0091$; OR 2.99), which was verified in the subsequent multivariate analysis with a p-value of 0.0475 . This is in accordance with the findings of Lewis et al. [14] and Pavlotsky et al. [15], who refer to the importance of tinea pedis in relapse of erysipelas.

Bacterial colonization of a leg affected by erysipelas has been subject of various studies. Generally, $\beta$-hemolytic streptococci are regarded to be responsible for erysipelas [6]. Therefore, Chartier et al. [16] examined the suspected entry sites for streptococci and found positive results in $26.9 \%$ of the cases. Lazzarini et al. analyzed the microbial colonization of wounds on the ipsilateral leg and found Staphylococcus aureus $(28.26 \%)$ and $\beta$-hemolytic streptococci $(11.96 \%)$ as the most frequent pathogens [17]. Jorup-Rönström [18] and Eriksson et al. [19] also found Staphylococcus aureus more common within the erysipelas collective.

In this study the two-sided Fisher exact test revealed a positive significant association between erysipelas and Staphylococcus aureus, coagulase-negative staphylococci, Enterococcus faecalis, Enterococcus faecium and $\beta$-hemolytic streptococci. In this respect Staphylococcus aureus, coagulase-negative staphylococci and Enterococcus faecalis were even shown to be highly significant, while $\beta$-hemolytic streptococci were significant. The result of multivariate analysis taking into account interdigital tinea pedis showed similar results. Only Staphylococcus aureus reached a p-value lower than 0.05 .

Of particular interest is the fact that the non-pathogenic aerobic bacteria were significantly negatively associated with erysipelas in both bivariate and multivariate analysis $\left(\mathrm{OR}_{\text {bivariate }}\right.$ 0.10 and $\mathrm{OR}_{\text {multivariate }} 0.25$ ), which means that they were found much more often in the control group than in the erysipelas collective. Possibly, physiological or non-pathogenic mixed flora as found in the oral cavity or the gastrointestinal tract may also provide a protective effect in the toe web. 
In this study we tried to minimize statistical bias and confounders for example by analyzing microbiological smears without diagnosis and by using additional multivariate logistic regression analysis. However, the fact that different persons performed the $\mathrm{KOH}$ examination might have resulted in a potential statistical bias. Moreover, data of the retrospective group might contain errors since they were based on the information taken from the patients' files exclusively. Considering the advanced age of patients included in our study collective, it remains uncertain in how far this is representative for the total population, especially as regards co-morbidities and immune status.

\section{Conclusion}

Quintessence of this study is that $\beta$-hemolytic streptococci, the main pathogen of erysipelas [5], are uncommonly found as an isolated organism in the toe web. It seems that the presence of Staphylococcus aureus and Enterococcus is of far more importance. Even though in multivariate analysis Enterococcus faecalis narrowly missed the significance threshold, enterococci should be included in diagnostic procedures since more and more frequently they are found to be responsible for skin infections [20].

This study indicates that interdigital tinea pedis plays an important role in the recurrence of erysipelas of the leg. As long as results of multi-center studies are missing, it remains to be seen whether this is due to a destruction of the skin barrier (maceration of the toe web) serving as entrance for potential pathogens and/or whether this environment favors colonization and growth of bacteria [21, 22].

According to current data every patient with erysipelas of the leg should undergo extensive diagnostic investigation including $\mathrm{KOH}$ examination and thorough microbiological analysis of the toe web. Positive test results should be followed by corresponding treatment in order to prevent relapse [23-25].

\section{Correspondence to}

Prof. Dr. med. Julia Welzel

Department of Dermatology

Klinikum Augsburg

Sauerbruchstraße 6

86179 Augsburg, Germany

E-mail: julia.welzel@klinikum-augsburg.de

\section{References}

1 Chartier C, Grosshans E. Erysipelas: an update. Int J Dermatol 1996; 35: 779-81.

2 http://www.gbe-bund.de/gbe10/i?i=475D (02.08.2013).

3 http://www.gbe-bund.de/gbe10/i?i=550D (02.08.2013).
4 Smolle J, Kahofer P, Pfaffentaler E et al. Risikofaktoren für das Auftreten von lokalen Komplikationen beim Erysipel. Hautarzt 2000; 51: 14-8.

5 Plewig G, Landthaler M, Burgdorf WHC et al. Braun-Falco's Dermatologie, Venerologie und Allergologie, 6. Auflage, Springer Medizin Verlag, Heidelberg 2012: 149-52, 243-59, 1086-8, 1103-13.

6 Rothmund G, Sattler EC, Kaestle R et al. Confocal laser scanning microscopy as a new valuable tool in the diagnosis of onychomycosis - comparison of six diagnostic methods. Mycoses 2013; 56: 47-55.

7 http:// flexikon.doccheck.com/de/Spezifität (04.08.2013).

8 http://flexikon.doccheck.com/de/Sensitivität (04.08.2013).

9 Weiß C. Basiswissen Medizinische Statistik, 5. Auflage, Springer Medizin Verlag, Heidelberg 2010: 19-76, 112-118, 177-192, 208-210, 287-91.

$10 \mathrm{http} / /$ www.bmj.com/about-bmj/resources-readers/publications/statistics-square-one/9-exact-probability-test (27.09.2013).

11 Roujeau JC, Sigurgeirsson B, Korting HC et al. Chronic dermatomycoses of the foot as risk factors for acute bacterial cellulitis of the leg: a case-control study. Dermatology 2004; 209: 301-7.

12 Roldan YB, Mata-Essayag S, Hartung C. Erysipelas and tinea pedis. Mycoses 2000; 43: 181-3.

13 Björnsdóttir S, Gottfredsson M, Thórisdóttir AS et al. Risk factors for acute cellulitis of the lower limb: a prospective case-control study. Clin Infect Dis. 2005; 41: 1416-22.

14 Lewis SD, Peter GS, Gómez-Marín O et al. Risk factors for recurrent lower extremity cellulitis in a U.S. Veterans Medical Center population. Am J Med Sci 2006; 332: 304-7.

15 Pavlotsky F, Amrani S, Trau H. Risikofaktoren für Rezidiveryipele. J Dtsch Dermatol Ges 2004; 2: 89-95.

16 Chartier C, Grosshans E. Erysipelas. Int J Dermatol 1990; 29: 459-67.

17 Lazzarini L, Conti E, Tositti G et al. Erysipelas and cellulitis: clinical and microbiological spectrum in an Italian tertiary care hospital. J Infec 2005; 51: 383-9.

18 Jorup-Rönström C. Epidemiological, bacteriological and complicating features of erysipelas. Scan J Infect Dis. 1986; 18: 519-24.

19 Eriksson B, Jorup-Rönström C, Karkkonen K et al. Erysipelas: Clinical and Bacteriologic Spectrum and Serological Aspects. Clin Infect Dis 1996; 23: 1091-8.

20 Nataraj G, Baveja S. Cutaneous bacterial infections: Changing trends in bacterial resistance. Indian J Dermatol Venereol and Leprol 2003; 69: 375-6.

21 Kates SG, Nordstrom KM, McGinley KJ et al. Microbial ecology of interdigital infections of toe web spaces. J Am Acad Dermatol 1990; 22: 578-82.

22 Leyden JJ, Kligman AM. Interdigital athlete's foot. The interaction of dermatophytes and resident bacteria. Arch Dermatol 1978; 114: 1466-72.

23 Kremer M, Zuckerman R, Avraham Z, Raz R. Long-term antimicrobial therapy in the prevention of recurrent soft-tissue infections. J Infect 1991; 22: 37-40.

24 Thomas KS, Crook AM, Nunn AJ et al. U.K. Dermatology Clinical Trials Network's PATCH I Trial Team. Penicillin to prevent recurrent leg cellulitis. N Engl J Med 2013; 368: 1695-703. Leclerc S, Teixeira A, Mahé E et al. Recurrent erysipelas: 47 cases. Dermatology 2007; 214: 52-7. 